\title{
Treatment of Capillary Hemangioblastoma in Von Hippel-Lindau Disease: Case Report and Online Survey of Treatment Preferences among Experts
}

\author{
Carlos Andrés Valdés-Lara Jose Arturo Oyervides-Alvarado \\ Juan Manuel Elizondo-Camacho Dhariana Acón-Ramírez \\ Jose Gerardo García-Aguirre \\ Retina and Vitreous Department, Asociación para Evitar la Ceguera en México IAP, \\ Hospital Dr. Luis Sánchez Bulnes, Mexico City, Mexico
}

\section{Keywords}

Von Hippel-Lindau disease · Capillary hemangioblastoma · Treatment · Online survey

\begin{abstract}
This case report aims to describe the diagnosis, treatment, and evolution of bilateral, asymmetrical retinal capillary hemangioblastomas treated with argon laser and intravitreal anti-vascular endothelial growth factor and also reports the results of an online survey of treatment preferences among retina and vitreous specialists. A previously healthy 23 -year-old female presented to our Retina Department complaining of progressive visual loss in her right eye. Visual acuity at admission was 20/300 in her right eye and 20/20 in her left eye. Anterior segment findings were unremarkable and fundoscopy revealed the presence of retinal capillary hemangioblastomas in both eyes. In the right eye, the hemangioblastoma was associated with pronounced exudation and macular edema; in the left eye, the lesion was quiescent. After a complete anamnesis and genetic counseling, Von Hippel-Lindau disease was diagnosed. Treatment with laser photocoagulation was performed on both eyes. One dose of $0.5 \mathrm{mg}$ intravitreal ranibizumab was applied to the right eye. Two months after treatment, the right eye
\end{abstract}


demonstrated improved visual acuity (20/100). Moreover, an important decrease in tumor dimensions and a reduction of vessel tortuosity was seen in both eyes. At 18 months of followup, the patient maintains a good visual acuity without recurrence of the treated tumors. Laser treatment should be considered as the primary treatment option for patients with capillary hemangioblastomas with and without exudation and can be combined with intravitreal antiangiogenics if exudation is significant. Inactive smaller lesions without exudation are likely to have an excellent response to laser treatment alone. Management should be individualized since no consensus between experts has been reached.

\section{Introduction}

Von Hippel-Lindau (VHL) disease is a rare multisystemic neoplastic syndrome arising from germline mutations in the VHL tumor suppressor gene. This gene is involved in the regulation of hypoxia-inducible factor, which in turn enhances glucose uptake and increases expressions of angiogenic, growth, and mitogenic factors (vascular endothelial growth factor [VEGF], platelet-derived growth factor-b, and transforming growth factor- $\alpha$ ) [1].

The incidence rate of VHL disease is approximately 1 in 36,000 live births; it has an autosomal dominant hereditary pattern and a penetrance of over $90 \%$ by 65 years of age. Cardinal manifestations include retinal hemangioblastomas (RHs), spinal cord and brain hemangioblastomas, renal cell carcinomas, pheochromocytomas, renal and pancreatic cysts, endolymphatic sac tumors, epididymis and broad ligament cystadenomas, and pancreatic neuroendocrine tumors [1-3].

RHs are benign neoplasms with vascular characteristics, which originate from the neurosensory retina or optic disc. They typically occur in the setting of VHL disease, although they can be isolated. Small RHs are round and flat but, as they increase in diameter, they often acquire a globular appearance. Characteristic feeding and drainage vessels become progressively enlarged and tortuous as the tumor grows. RHs are typically asymptomatic at early stages; nevertheless, in advanced stages, these tumors turn exudative, resulting in diffuse retinal edema with hard exudates which often form a ring or "circinate" pattern around the vascular lesion. Vision loss is usually caused by exudation affecting the macula, glial proliferation or posterior hyaloid contraction related to the tumor and, in severe cases, tractional and/or exudative retinal detachment [4-6]. Intraocular pressure elevation secondary to iris neovascularization and neovascular glaucoma has been described [4, 5]. Diagnosis of VHL disease is based on clinical criteria and confirmed by molecular testing $[5,6]$.

\section{Case Report}

A previously healthy 23-year-old Hispanic female from a community in the South of Mexico presented at the Retina and Vitreous Department of our hospital with a history of 4 weeks of progressive, nonpainful decrease of vision in her right eye. At interrogatory, the family medical history of brain and renal tumors was positive in 3 generations. Personal past medical history was denied. At examination, visual acuity was 20/300 in the right eye and 20/20 in the left eye, with no significant refractive error. Intraocular pressure was 12 and $14 \mathrm{~mm} \mathrm{Hg}$, respectively. Anterior segment examination was unremarkable. Ocular funduscopy of the right eye showed an orange, elevated, well-demarcated lesion of 3 disc diameters with tortuous feeding vessels at the end of the superior temporal arcade. Multiple hard exudates were 
present in a circinate distribution around the tumor, affecting the posterior pole and the superior-nasal quadrant. In the temporal-inferior periphery of the same eye, another small exudative lesion of 1 disc diameter was detected (Fig. 1a). In the left eye, within the temporal inferior quadrant, a 1-disc diameter tumor with tortuous feeder vessels and without exudation was found (Fig. 1b).

Fluorescein angiography of the right eye showed early hyperfluorescence of the tumor, which increased throughout the angiogram phases. Leakage and macular edema were found (Fig. 2a). Left eye angiography showed hyperfluorescence of the tumor without leakage (Fig. 2b). On optical coherence tomography (OCT), the tumor appeared as a dense hyperreflective mass with posterior shadowing located in the inner layers of the retina. Intraretinal cysts and diffuse macular edema were documented in the right eye (Fig. 2c). Macular OCT of the left eye was normal (Fig. 2d). VHL disease diagnosis was made after a thorough clinical examination, genetic and internal medicine counseling, and complementary exams, including a brain magnetic resonance imaging and abdominal ultrasound; all of these studies were within normal parameters. Genetic testing was indicated but not performed because of the patient's economic limitations. Other causes of vascular retinal abnormalities were ruled out, such as vasoproliferative tumor, cavernous retinal hemangioma, and Wyburn-Mason disease.

Argon laser treatment (PUREPOINT ${ }^{\circledR}$, Alcon, Dallas Fort Worth, TX, USA) was performed to all tumors in both eyes. Initial parameters used included a spot size of $400 \mu \mathrm{m}$, exposition time of $400 \mathrm{~ms}$, and power of $200 \mathrm{~mJ}$. Laser was applied directly to the tumor until the entire lesion was covered. A total of 8 laser sessions, with 3-week separation intervals, were applied to the main lesion of the right eye and 3 laser sessions using the same parameters were employed in the left eye. Only 1 dose of $0.5 \mathrm{mg}$ intravitreal ranibizumab was injected in the right eye during the first month. Two months after laser treatment, there was improvement in visual acuity from 20/300 to 20/100 in the right eye, as well as regression of the tumor and its feeding vessels (Fig. 3a). In the left eye, the lesion had a significant decrease in size (Fig. 3b).

At the 18-month follow-up visit, a complete clinical examination and fundoscopy were performed; no other laboratory or imaging exams were required. Visual acuity was 20/80 in the right eye and 20/20 in the left eye. A complete regression of the lesions in the right eye can be observed as chorioretinal scar, and no clinically significant exudation was present (Fig. $3 c$ ). In the left eye, only a chorioretinal scar is evident in the area where the vascular tumor used to be (Fig. 3d). Macular OCT of the right eye revealed absence of subretinal or intraretinal fluid (Fig. 3e). No other lesion was found. The patient continues with close medical follow-up with an ophthalmologist and an internal medicine specialist. Family counseling was offered; 3 other members have been diagnosed with VHL disease, including a younger sister.

\section{Discussion}

We present a case of a previously undiagnosed patient with VHL disease with bilateral RHs in different clinical stages. The treatment we provided was successful and not only the visual acuity of the patient improved; now, other family members are well aware of their illness and are receiving medical care. An online survey of retinal specialists was conducted, and we concluded that treatment is controversial and should be individualized depending on the patient's characteristics (Fig. 4). One of the limitations of this case report is that the patient was economically limited; genetic testing was indicated but not carried out, and follow-up studies, such as macular OCT, were not performed on all visits. 
Currently, there is no consensus regarding the management of RHs. A wide variety of therapies have been used alone or in combination with mixed results. Before vision loss occurs, small tumors can be easily diminished with minimal risks; however, spontaneous regression has been reported [7]. On the other hand, larger lesions can be much more difficult to treat, and damage induced by treatment often leads to a variable degree of exudation and sometimes to a significant inflammatory response that can threaten vision $[8,9]$.

Several ablative modalities have been used for the treatment of extrapapillary RHs, including thermal laser photocoagulation, cryotherapy, radiation (including brachytherapy, external beam radiation, and proton beam radiation), photodynamic therapy, and transpupillary thermotherapy $[8,9]$. Other treatments, such as surgical removal of large RHs and pharmacotherapy (intravitreal administration of VEGF antagonists) to mitigate tumor exudation, have been employed with variable results $[8,9]$.

A recent retrospective study performed by Krivosic et al. [9] evaluated the efficacy and limits of laser photocoagulation in patients with VHL disease and RHs of various sizes. A total of 176 patients were included, but only 74 patients (with 304 RHs in 100 eyes) were treated mainly with laser photocoagulation. Inactivation of RHs was achieved using direct green laser with long duration burns (0.1-0.7 s). In the absence of tractional retinal detachment, laser photocoagulation achieved the inactivation of most RHs up to 3 disc diameters, even when subretinal fluid was present. Laser photocoagulation as a single treatment inactivated $100 \%$ of the RHs up to 1 disc diameter and up to $73 \%$ of larger RHs. In these cases, additional cryotherapy increased the inactivation rate to $94 \%$ [9].

Because of the controversy surrounding the most appropriate treatment for our patient, we conducted an electronic online survey with the objective to inquire the experts' opinion. In this document, the clinical summary of the patient was included describing her visual acuity at admission, her family and personal history highlights, and a series of images, including clinical photographs, fluorescein angiography, and OCT. The survey was sent via e-Mail to 65 Spanish-speaking vitreoretinal surgeons. A 66\% response rate was obtained. Physicians were originally from Mexico (60\%) and other Spanish-speaking countries (16\%; including Spain, Colombia, Argentina, Costa Rica, Puerto Rico, and Peru), and 23\% did not provide this information.

When questioned about the initial management of the main lesion in the right eye, 35\% of specialists chose to start with a combination of laser photocoagulation plus intravitreal antiangiogenic, $16 \%$ with antiangiogenic monotherapy, $14 \%$ with a combination of antiangiogenic and photodynamic therapy, $14 \%$ with 3 or more combined therapies, and $12 \%$ with photocoagulation monotherapy (Fig. 5). Regarding laser photocoagulation parameters, answers were widely diverse when asked about the exposure time, power, and way to direct the laser probe; therefore, a conclusion about laser parameters was not reached.

While $72 \%$ of the participants agreed on the need to start immediate treatment on the small exudative peripheral lesion of the right eye, treatment of the left eye injury was controversial. Most specialists argued that since the left eye had a preserved visual acuity and did not show clinical or angiographic data of active exudation, maintaining clinical observation (some with additional maneuvers, such as fluorescein angiography or clinical photographs) was the best option. However, $28 \%$ chose immediate treatment, arguing that smaller lesions are easier to treat with photocoagulation (Fig. 6).

Larger lesions could be much more difficult to destroy, and injury caused by treatment frequently results in an unpredictable exudative response that can occasionally cause retinal detachment and vision loss. Therefore, a key feature of RH management is to identify tumors as early as possible in order to offer appropriate treatment. 
Extrapapillary RHs up to $1.5 \mathrm{~mm}$ (1 disc diameter) in diameter can consistently be destroyed using thermal laser photocoagulation across 1 or more sessions. RH between 1.5 and $4.5 \mathrm{~mm}$ in diameter are more difficult to extinguish using thermal laser photocoagulation. Commonly, multiple sessions are needed to achieve success, and some lesions persist after multiple treatment sessions. Additionally, cryotherapy is an option for RHs in this size range; it could be used as treatment for refractory tumors to previous laser photocoagulation or as first-line therapy. RHs greater than $4.5 \mathrm{~mm}$ in diameter represent a more difficult challenge, with low rates of success and higher risks of treatment. Thermal laser photocoagulation and photodynamic therapy are usually ineffective for these tumors, and there is no consensus on how these lesions should be treated.

Intravitreal VEGF antagonists could be used in an attempt to control exudation in cases where ablative therapy is not possible or has failed. Some eyes can exhibit at least modest improvement in intraretinal edema or subretinal fluid.

Currently, there is no consensus regarding the management of RHs. In this case, we performed combined therapy for the right eye; laser targeting tumor regression and antiangiogenic drug to aim for macular edema. We decided to treat the peripheral tumor in the left eye with laser because smaller tumors are easier to destroy with laser and, on the other hand, the patient had an aggressive evolution with visual acuity compromise in the other eye.

\section{Conclusions}

The clinical picture of RHs is widely heterogeneous and their behavior might be unpredictable. There is no consensus for treatment, even among the retina and vitreous specialists questioned. We present a case where the patient had an excellent clinical evolution with the selected treatment. Laser photocoagulation combined with intravitreal antiangiogenic therapy might be an appropriate initial treatment for large, exudative RHs. Inactive smaller lesions without exudation are likely to have an excellent response to laser treatment alone. Management of these patients continues to be challenging, and treatment must be individualized for each patient.

\section{Acknowledgement}

We acknowledge the help of Roberto González Salinas and Laura Alejandra GonzálezDíbildox.

\section{Statement of Ethics}

Written informed consent was obtained from the patient.

\section{Disclosure Statement}

The authors declare no conflict of interest. 


\section{Case Reports in Ophthalmology}

Valdés-Lara et al.: Treatment of Capillary Hemangioblastoma in Von Hippel-Lindau Disease: Case Report and Online Survey of Treatment Preferences among Experts

\section{Funding Sources}

No financial support was received.

\section{Author Contributions}

Conceived and designed the analysis: Jose Gerardo García-Aguirre, Jose Arturo OyervidesAlvarado.

Collected the data: Jose Arturo Oyervides-Alvarado.

Contributed data or analysis tools: Juan Manuel Elizondo-Camacho, Dhariana Acón-Ramírez.

Performed the analysis: Carlos Andrés Valdés-Lara.

Wrote the paper: Jose Arturo Oyervides-Alvarado, Carlos Andrés Valdés-Lara.

\section{References}

1 Lonser RR, Glenn GM, Walther M, Chew EY, Libutti SK, Linehan WM, et al. von Hippel-Lindau disease. Lancet. 2003 Jun;361(9374):2059-67.

2 Maher ER, Iselius L, Yates JR, Littler M, Benjamin C, Harris R, et al. Von Hippel-Lindau disease: a genetic study. J Med Genet. 1991 Jul;28(7):443-7.

3 Schmid S, Gillessen S, Binet I, Brändle M, Engeler D, Greiner J, et al. Management of von Hippel-Lindau disease: an interdisciplinary review. Oncol Res Treat. 2014;37(12):761-71.

4 Chew E, Schachat AP. Capillary hemangioblastoma of the retina and von Hippel-Lindau Disease. In: Ryan SJ, Wilkinson CP, Schachat AP, Hinton DR, Wiedemann P, editors. Retina. 5th ed. Philadelphia: Saunders; 2013. pp. 2427-35.

5 Singh AD, Shields CL, Shields JA. von Hippel-Lindau disease. Surv Ophthalmol. 2001 Sep-Oct;46(2):117-42.

6 Wong WT, Agrón E, Coleman HR, Tran T, Reed GF, Csaky K, et al. Clinical characterization of retinal capillary hemangioblastomas in a large population of patients with von Hippel-Lindau disease. Ophthalmology. 2008 Jan;115(1):181-8.

7 Whitson JT, Welch RB, Green WR. Von Hippel-Lindau disease: case report of a patient with spontaneous regression of a retinal angioma. Retina. 1986;6(4):253-9.

8 Kim H, Yi JH, Kwon HJ, Lee CS, Lee SC. Therapeutic outcomes of retinal hemangioblastomas. Retina. 2014 Dec;34(12):2479-86.

9 Krivosic V, Kamami-Levy C, Jacob J, Richard S, Tadayoni R, Gaudric A. Laser Photocoagulation for Peripheral Retinal Capillary Hemangioblastoma in von Hippel-Lindau Disease. Ophthalmol Retina. 2017 JanFeb;1(1):59-67. 


\section{Case Reports in Ophthalmology}
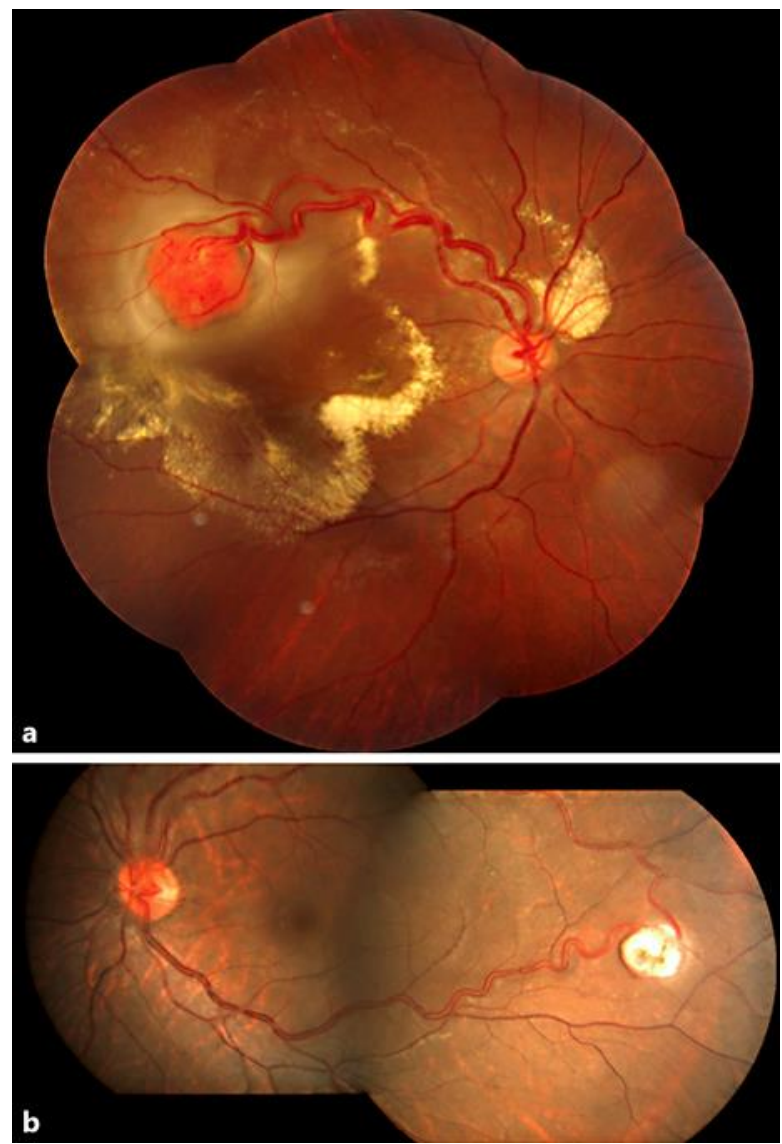

Fig. 1. a Composite retinal fundus image of the right eye. One lesion with globular appearance, dilated feeding vessels, and abundant exudation is observed at the superior-temporal vascular arcade. $\mathbf{b}$ Composite retinal fundus image of the left eye. One lesion with globular appearance, dilated feeding vessels, and absence of exudation is observed at the inferior-temporal periphery. 


\section{Case Reports in Ophthalmology}
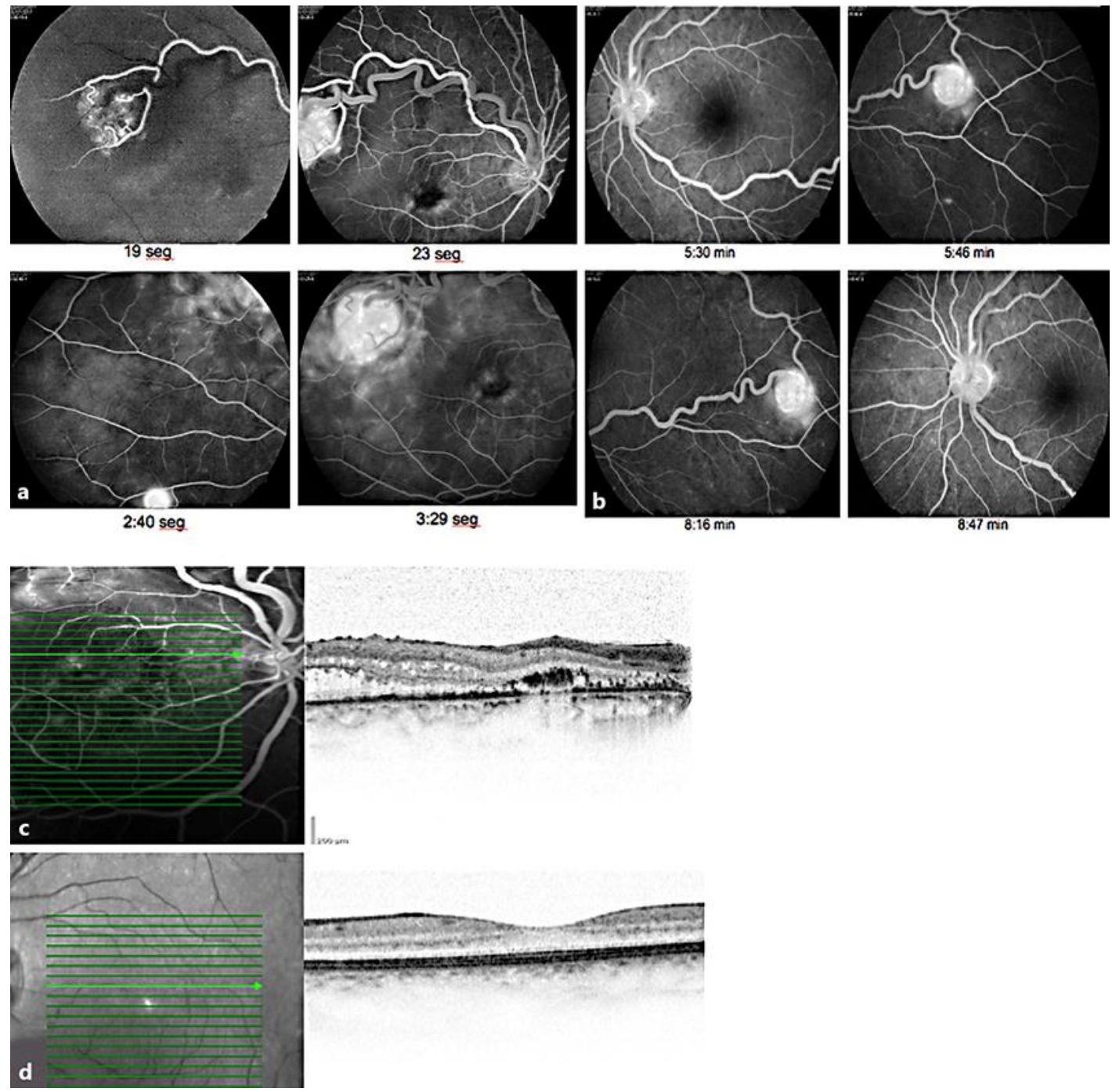

Fig. 2. a Fluorescein angiography of the right eye. Lesion located in the superior-temporal vascular arcade; note the dilated feeding vessels in early phases and abundant exudation in late phases. b Fluorescein angiography of the left eye. Lesion with globular appearance, dilated feeding vessels, and absence of exudation in all phases. c Macular OCT of the right eye; diffuse macular edema with hyperreflective foci in the outer layers corresponding with hard exudates. $\mathbf{d}$ Macular OCT of the left eye; absence of pathological findings in the macular area. 


\section{Case Reports in Ophthalmology}
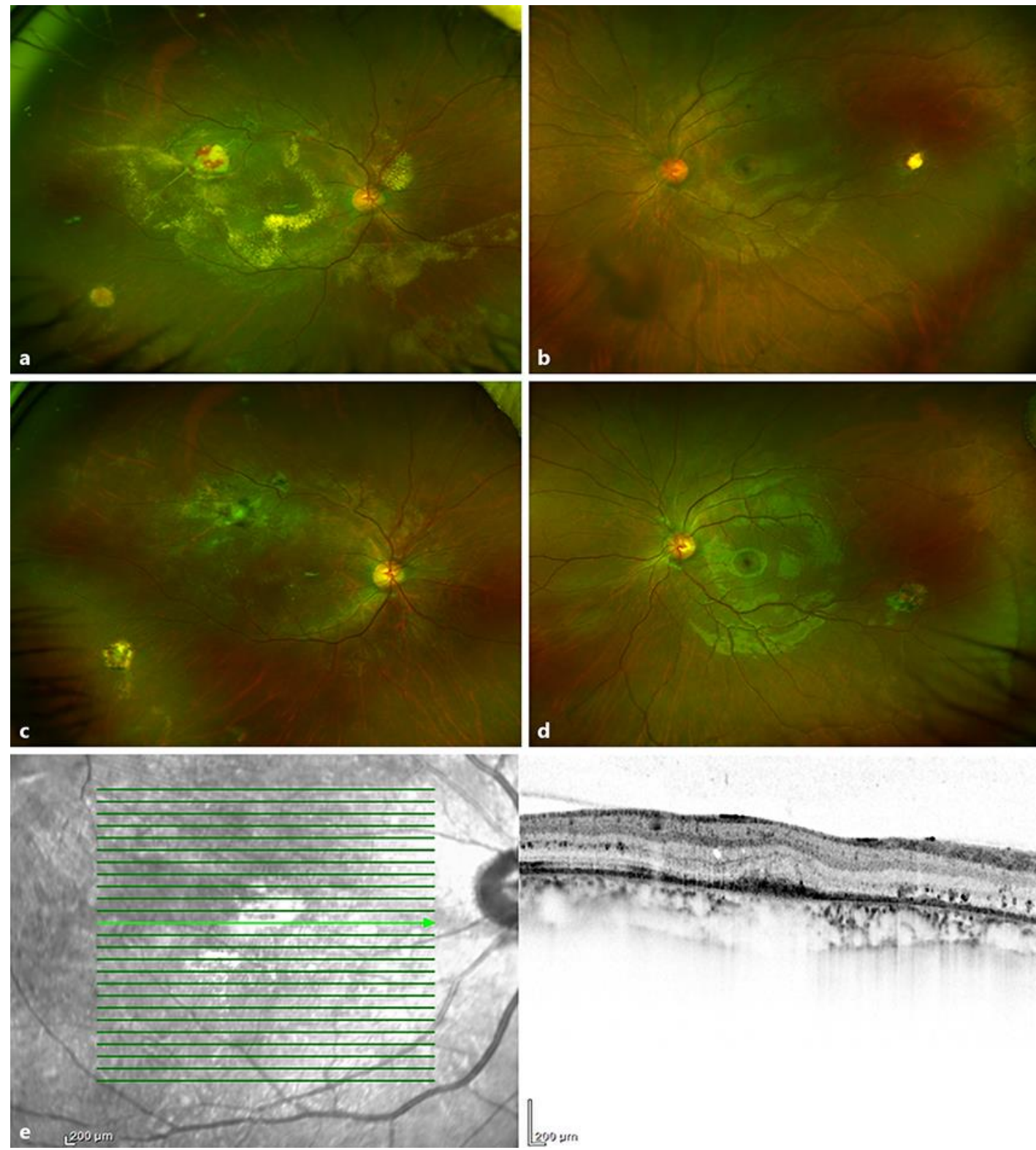

Fig. 3. a, b Wide-field fundus photographs, 2 months after treatment. Areas of sclerosis start to appear in the vascular lesions of both eyes. A decrease in thickness of the feeding vessels can also be appreciated. c, $\mathbf{d}$ Wide-field fundus photographs at 18-month follow-up; complete resolution of the lesions in both eyes as chorioretinal scars and no exudation. e Macular OCT of the right eye at 18-month follow-up; abnormal architecture of inner and outer retinal layers, loss of foveal depression, and absence of intraretinal and subretinal fluid. 


\section{Case Reports in Ophthalmology}

\begin{tabular}{l|l}
\hline Case Rep Ophthalmol 2020;11:37-47 \\
\hline DOI: 10.1159/000504430 & $\begin{array}{l}\text { @ 2020 The Author(s). Published by S. Karger AG, Basel } \\
\text { www.karger.com/cop }\end{array}$ \\
\hline
\end{tabular}

Valdés-Lara et al:: Treatment of Capillary Hemangioblastoma in Von Hippel-Lindau Disease: Case Report and Online Survey of Treatment Preferences among Experts
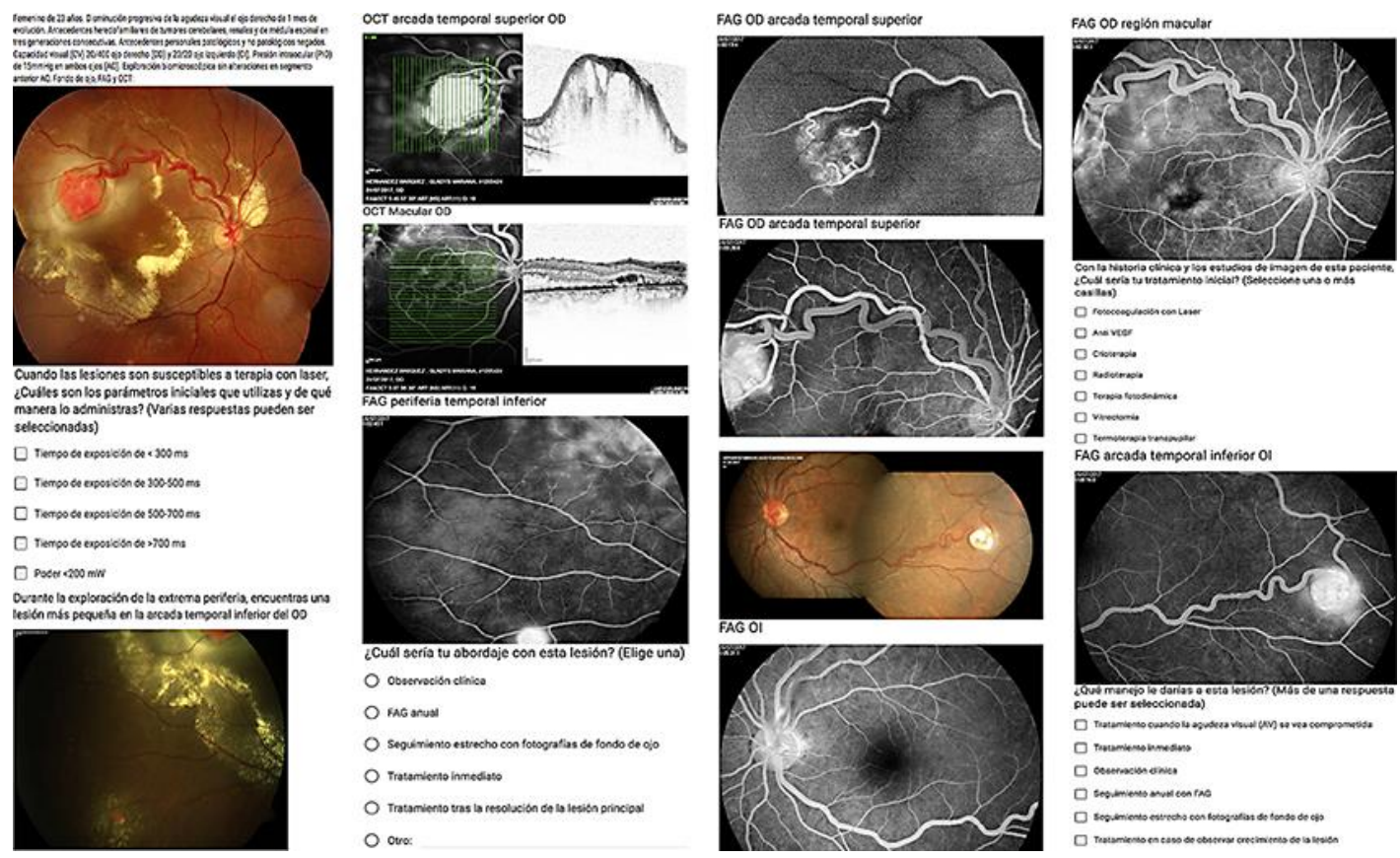

Fig. 4. Online survey. The online survey included a complete clinical case description, funduscopic, macular OCT, and fluorescein angiographic images of both eyes, mixed with multiple choice and open questions.

\section{Main Lesion Right Eye}

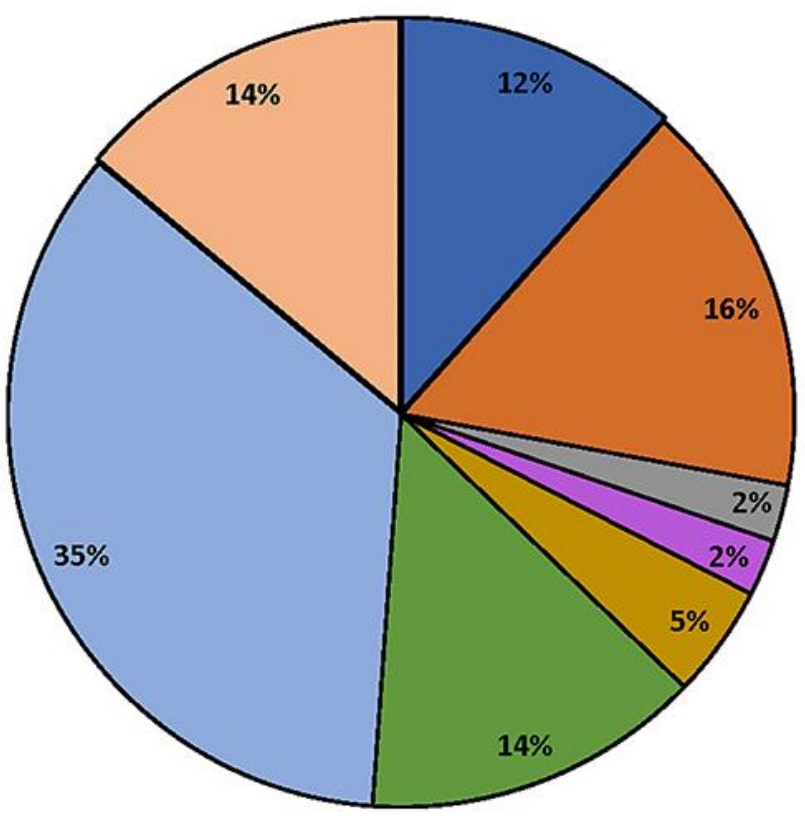

\section{口Photocoagulation monotherapy}

$\square$ Antiangiogenic monotherapy

$\square$ Photodynamic monotherapy

$\square$ Photocoagulation plus photodynamic

$\square$ Antiangiogenic plus cryotherapy

$\square$ Antiangiogenic plus photodynamic

$\square$ Photocoagulation plus antiangiogenic

$\square$ Three or more combined therapies

Fig. 5. Electronic survey results: preferred treatment for the right eye. 


\section{Left Eye Approach}

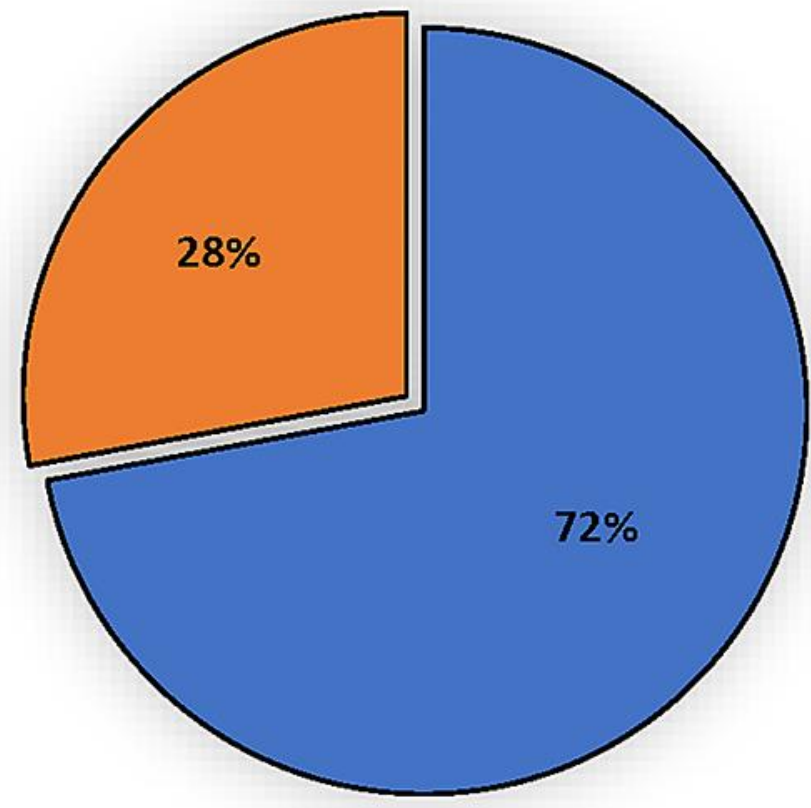

$\square$ Clinical observation $\square$ Immediate treatment

Fig. 6. Electronic survey results: suggested management for the left eye. 